\title{
Effects of geometrical characteristics on defect distributions in alloy components produced by selective laser melting
}

\author{
Yao Cai', Tao Lu', Gui-dian Ma², Wang Lí2, *Ye Pan', **Hui Ding1 \\ 1. School of Materials Science and Engineering, Southeast University, Jiangsu Key Laboratory of Advanced Metallic Materials, Nanjing 211189, China \\ 2. Nanjing Chamlion Laser Technology Co., Ltd, Nanjing 210039, China
}

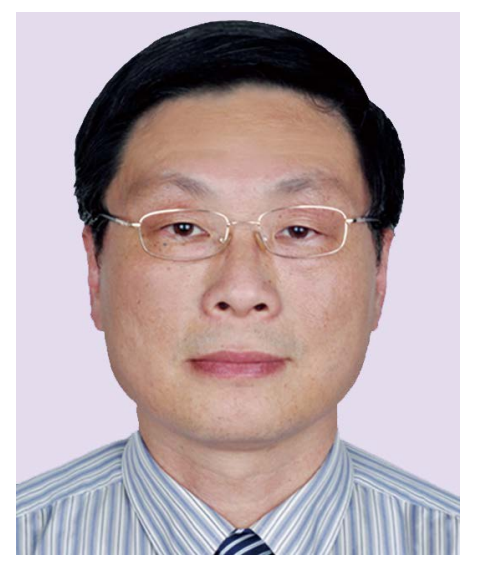

*Ye Pan

Ph.D., Professor. His research interests mainly focus on the fabrication and properties of advanced metallic materials, solidification and microstructure controlling of metals, metallic glass and high entropy alloys, etc. To date, he has published more than 160 academic papers in international and domestic journals. $\mathrm{He}$ is an executive member of the Foundry Institution of Chinese Mechanical Engineering Society.

E-mail: panye@seu.edu.cn

**Hui Ding

E-mail: dinghui@seu.edu.cn

Received: 2021-03-16

Accepted: 2021-06-10
Abstract: Selective laser melting (SLM) has been applied to manufacture various alloy components with excellent properties, but its further application is restricted by the intrinsic defects. In this work, the internal defect distributions in samples of three alloys (316L stainless steel, AISi10Mg and Inconel 718) were investigated respectively, considering the effects of geometrical characteristics of the samples. The defects in the $316 \mathrm{~L}$ stainless steel sample tend to be formed densely in the central part with large wall thickness, indicating a strong sensitivity to heat accumulation. Contrarily, the Inconel 718 sample shows a higher relative density with homogeneous defect distribution, indicating better formability for the SLM process. For the AISi10Mg sample, the defect density keeps increasing as the deposition goes on. Typically, the defect density located at sample edges shows an abnormally high level comparing with the inner part, especially in the top sections of AISi10Mg and Inconel 718 samples. The results are helpful for the geometrical design, the adjustment of building orientation and the further optimization of process parameters in the SLM process.

Key words: selective laser melting; defect; 316L stainless steel; AISi10Mg; Inconel 718; geometrical characteristics; formability

CLC numbers: TG142.71/146.21/146.1 5; Document code: A;

Article ID: 1672-6421(2021)04-369-10

\section{Introduction}

Additive manufacturing (AM) is profoundly changing the traditional manufacturing industry. Among the additive manufacturing systems, selective laser melting (SLM) has developed into a mature forming process and has been applied in aerospace, automobile, medical treatment and other fields after decades of research ${ }^{[1,2]}$. To date, the industrial application of SLM is limited mainly due to the intrinsic defects in the formed parts ${ }^{[3]}$. The main cause for these defects lies in the extremely short interaction time between laser beam and alloy powder, and the metal materials continuously undergo the rapid heatingcooling process during the printing process ${ }^{[4]}$. Thus, defects are easily generated, such as cracks and pores, which deteriorate the forming quality. There are three main kinds of defects in SLM parts including gas pore, lack of fusion (LoF), and keyhole ${ }^{[5]}$. A gas pore defect is mainly caused by the inert gas in the building chamber or the gas remaining in the metal powder dissolved into the molten pool during the forming process that may be entrapped in the molten pool after solidification ${ }^{[3]}$. Lack of fusion is mainly caused by insufficient local heat input, while keyhole is due to the excessive local heat input ${ }^{[6]}$. For specific alloys, there are also material factors, such as the high viscosity of molten stainless steel and the oxidation of aluminum alloy, which may lead to gas pore defects ${ }^{[7,8]}$.

Previously, the research on SLM process mainly focused on the optimization of process parameters to eliminate defects. After decades of research, the optimal process 
parameters of several materials commonly used in SLM, such as $316 \mathrm{~L}$ stainless steel, AlSi10Mg and Inconel 718 as the common alloys in the engineering field, are basically determined, through which nearly full-dense parts can be fabricated. Liverani et al. ${ }^{[9]}$ concluded that the high relative density (over 99\%) of SLM 316L stainless steel parts can be obtained with the combination of laser power at $150 \mathrm{~W}$, and scanning speed of $700 \mathrm{~mm} \cdot \mathrm{s}^{-1}$ and hatch spacing at $70 \mu \mathrm{m}$. Kempen and Choi et al. ${ }^{[8,10]}$ determined the optimal process parameters of AlSi10Mg alloy and Inconel 718 alloy, respectively, and produced samples with density higher than $99 \%$.

A regular cubic/cylindrical sample is often used to investigate the optimization of process parameters, but the height and wall thickness are totally different in real components meeting the structural requirement. Thus, it is not reasonable enough to evaluate the internal defect situation in real components through the experience in the fabrication of regular samples ${ }^{[11]}$. Besides, in the practical application, it can be found that the defects cannot be completely eliminated which indicates that there still exist other factors affecting the formation and distribution of defects under the optimized process parameters ${ }^{[12]}$. Therefore, it is of great academic significance and practical application value to analyze the types and distribution of defects in the complexshaped component for accurately evaluating the forming ability of materials and predicting the possible positions of defects in parts to guide the structural design and further optimization of SLM. Therefore, in the present study, the testing samples with a specific shape were fabricated by SLM process, and the defect analysis for the SLM samples was conducted via micro-computed tomography (micro-CT) and metallography, respectively. The distributions of defects in the different sections and the entire samples were investigated to figure out the actual factors influencing defect distribution under the premise of process optimization.

\section{Experimental procedure}

\subsection{Materials and process parameters}

In this study, three types of commercial alloy powders $(316 \mathrm{~L}$ stainless steel, AlSi10Mg and Inconel718) were applied to fabricate experimental samples by a SLM280 machine via a SLM process. The process parameters used in this study, which have been previously optimized in industrial production, are listed in Table 1. High-purity argon (99.9999\%) was used to prevent oxidation in the working chamber. The laser moves through a meander or chessboard way as illustrated in Fig. 1(a) with a rotation angle of $67^{\circ}$ between adjacent layers. The shape and dimension of the samples are shown in Fig. 1(b). The shape was designed as a pentagonal prism because it contains quite a few features which may affect the distribution of defects, i.e., the building direction $Z$, horizontal plane $X$ - $Y$ with continuously changing width and horizontal and vertical edges.

Table 1: Optimized process parameters for three alloys

\begin{tabular}{|c|c|c|c|c|c|}
\hline Alloy & $\begin{array}{l}\text { Laser power } \\
\text { (W) }\end{array}$ & $\begin{array}{l}\text { Scanning speed } \\
\left(\mathrm{mm} \cdot \mathrm{s}^{-1}\right)\end{array}$ & $\begin{array}{l}\text { Hatch spacing } \\
\text { ( } \mu \mathrm{m})\end{array}$ & $\begin{array}{l}\text { Layer thickness } \\
\qquad(\mu \mathrm{m})\end{array}$ & Scanning strategy \\
\hline $316 \mathrm{~L}$ & 180 & 1,500 & 50 & 30 & $\begin{array}{l}\text { Chessboard } \\
(5 \mathrm{~mm} \times 5 \mathrm{~mm})\end{array}$ \\
\hline Inconel718 & 160 & 1,200 & 70 & 30 & $\begin{array}{l}\text { Chessboard } \\
(5 \mathrm{~mm} \times 5 \mathrm{~mm})\end{array}$ \\
\hline AISi10Mg & 350 & 1,650 & 130 & 30 & Meander \\
\hline
\end{tabular}

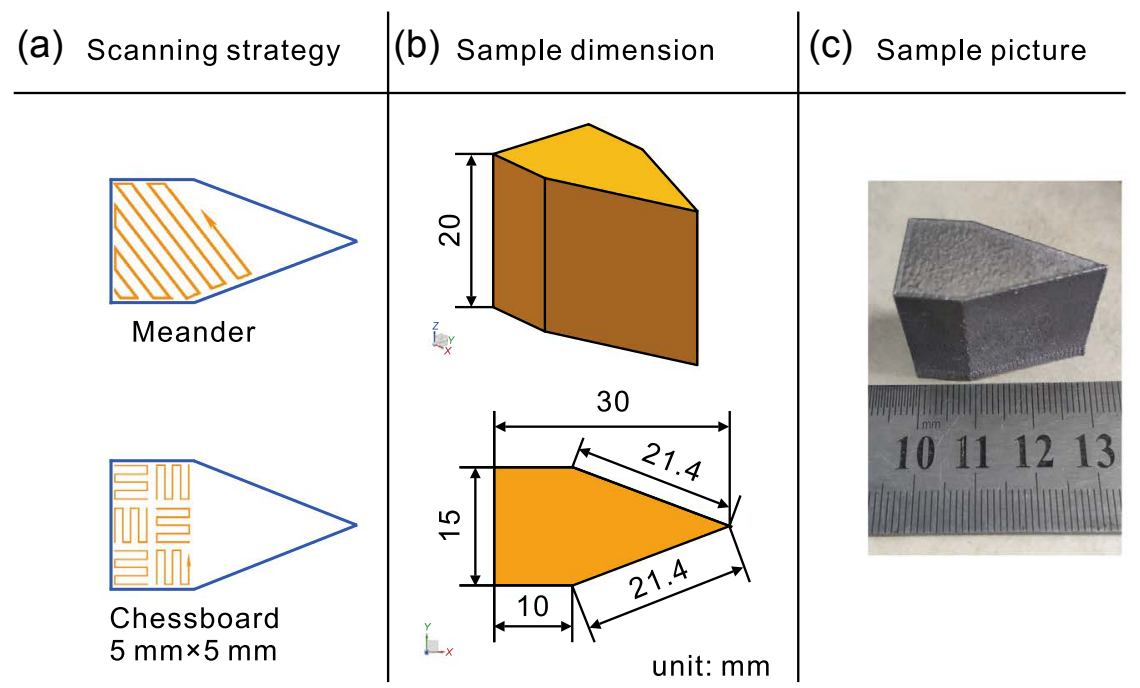

Fig. 1: Schematic illustration of scanning strategies (a), shape and dimension of samples (b), and picture of a sample (c) 


\subsection{Micro-CT}

Micro-CT tests were conducted with a YXLON FF35 industrial CT system. The spatial resolutions of the CT system, which can be interpreted as the minimum detectable size, are $25 \mu \mathrm{m}$ for $316 \mathrm{~L}$ stainless steel, $25 \mu \mathrm{m}$ for Inconel 718 and $7 \mu \mathrm{m}$ for AlSi10Mg. Defects with dimensions smaller than these resolutions will be analyzed by metallographic method. The image reconstruction and the analysis of the dataset were carried out using VG Studio MAX software. The volume and size distribution of the defects were calculated during the analysis.

\subsection{Metallography}

The samples prepared for metallography were fabricated on the same substrate in the same batch with the samples prepared for micro-CT. For optical microscopy, 24 cross sections in total were prepared via a standard metallographic procedure according to ASTM E3-11(2017). 2D images were then obtained using a CX40M optical microscope. Four vertical cross sections (marked as Section A, Section B, Section C and Section D) and four horizontal cross sections (marked as Section 1, Section 2, Section 3 and Section 4) of each alloy sample were obtained using wire electrical discharge machining (WEDM) in certain positions as shown in Fig. 2. Along the building direction $Z$, each cross section was divided into 20 areas and the height of the upper side of each small area is taken as the height of the whole area. In the horizontal direction, each section was divided into dozens of areas of $1 \mathrm{~mm} \times 1 \mathrm{~mm}$ and the small areas near the edge less than this size were calculated separately. Then, binarized images were created to calculate the defect area ratio (DAR) of each area using Image-Pro Plus software. Due to a small number of defects with large size (usually greater than $80 \mu \mathrm{m}$ ) in the samples, considering that CT testing has already been able to effectively detect such large holes and it is also required to remove outliers during the statistical calculation, such large defects should be ignored when calculating DAR. In general, defect sizes smaller than $80 \mu \mathrm{m}$ were analyzed quantitatively by metallography, while defect sizes larger than the spatial resolutions of the CT system were detected by micro-CT for visual judgment, both of which ensured a comprehensive investigation on the defect distributions of the samples.

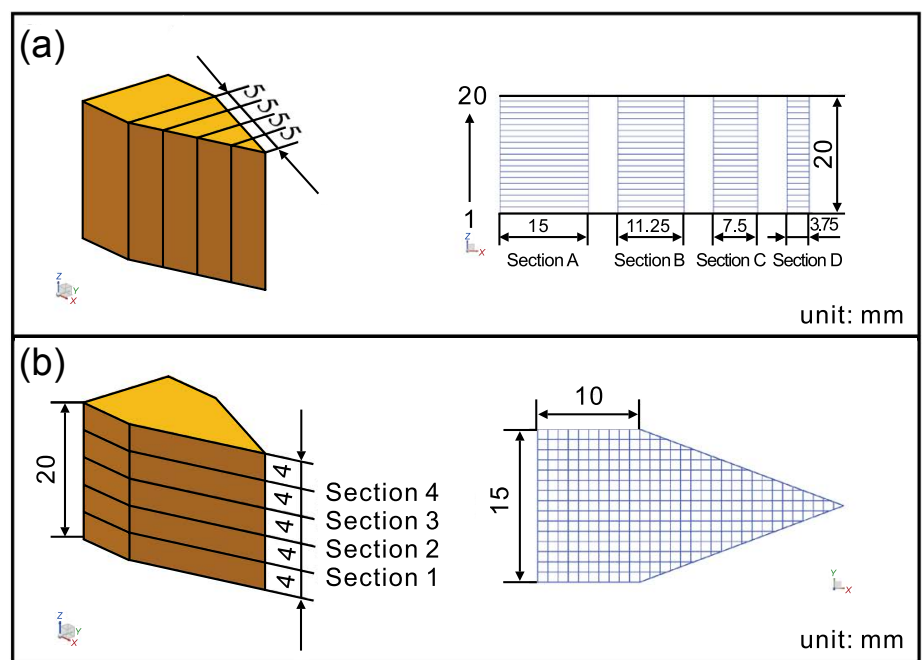

Fig. 2: Cutting method and cross sections division method: (a) cross section in vertical direction; (b) cross section in horizontal direction

\section{Results and discussion}

\subsection{Defect types}

Figure 3 reveals the morphology of defects inside the prepared samples with optical microscopy. Three types of defects can be observed in the polished surfaces of all samples, which are LoF and gas pore and keyhole. The gas pores exhibit a spherical shape with a diameter of less than $30 \mu \mathrm{m}$, while LoFs show an irregular shape with a much larger size. For keyholes, the size is usually between that of LoFs and gas pores, and an irregular shape with a sharp edge is observed. From the metallographic figures of horizontal and vertical sections, the distribution of all types of defects can be determined. Gas pores and keyholes are usually formed inside a single scan track, while LoFs are usually generated between neighboring scan tracks. Most gas pores are generated because the molten pool which solidifies rapidly can trap the gas locally during the SLM process ${ }^{[3]}$. As the cooling process goes on, the solubility of the gas in the molten pool decreases. Due to the extremely short solidification time, some of the gas has no time to escape, resulting in the formation of pores. Thus, the distribution of porosity can be considered as a reflection of the heating-cooling condition of the local molten pool ${ }^{[12]}$. Meanwhile, the existence of LoFs and keyholes indicates the non-uniform local heat input. As the heat input is insufficient, the incomplete melting of powder or loose overlapping of molten pools may cause LoFs ${ }^{[13]}$. In addition, the insufficient local heat input may lead to balling of the liquid phase which also causes LoFs. In contrast, the excessive local heat input leads to the evaporation of the liquid phase and forms a keyhole near the bottom of the melt pool after solidification. Specifically, for Al-based alloy, since the alloy powder inevitably contains a small amount of water vapor, the aluminum alloy is easily oxidized, leading to hydrogen reduction which causes the formation of gas pores and aluminum oxide ${ }^{[14,15]}$. Due to the extremely high melting point, the aluminum oxide is difficult to be completely melted by laser, which causes the decrease of the flowability of the liquid phase. As a result, lack of fusion defects are generated. It should be noted that the process parameters for each alloy are optimized to ensure an appropriate heat input locally and a global good bonding, so as to eliminate pore, keyhole and LoF defects as much as possible, but defects still cannot be completely avoided. It is reasonable that the complex heating-cooling condition of the SLM process is also relevant to the geometrical variation of the deposited part. Therefore, micro-CT and optical microscopy are applied to investigate the distribution of LoFs and porosity, respectively, considering the geometrical characteristics and deposition process of the component. 


\section{Unetched}

(a)

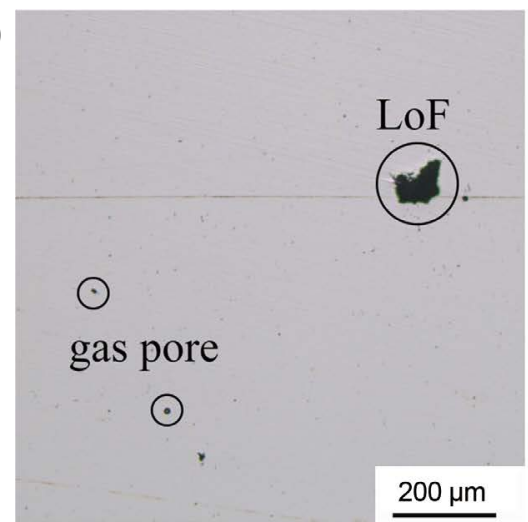

(b)

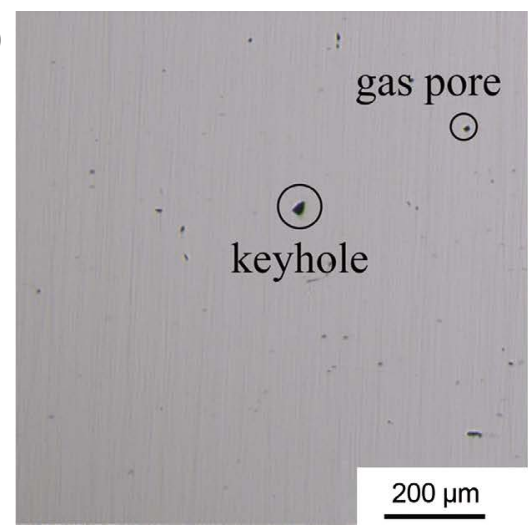

(c)

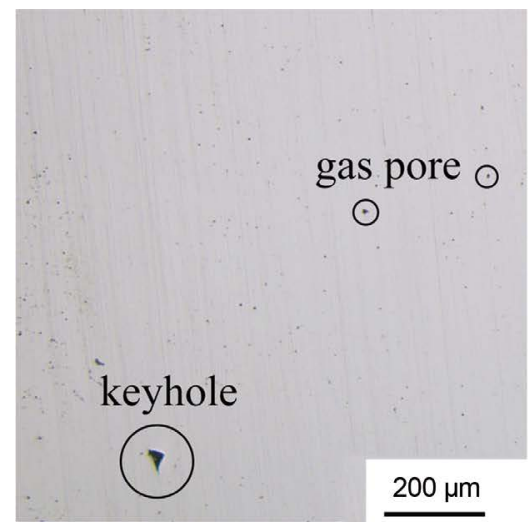

Horizontal
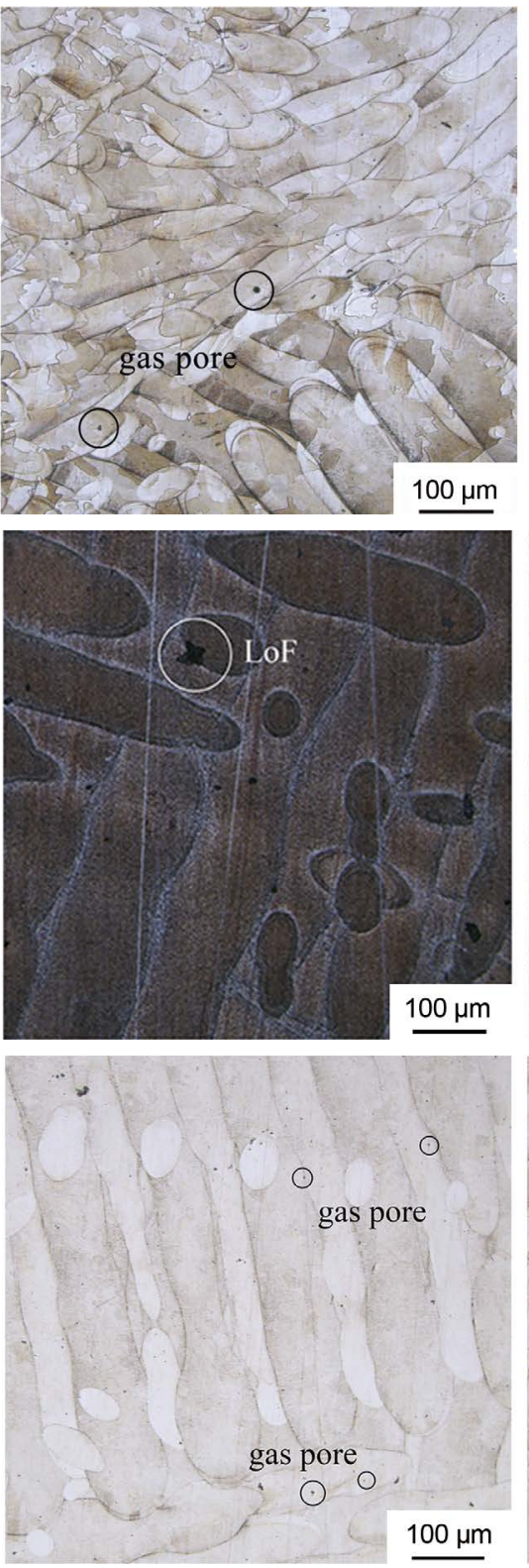

Vertical
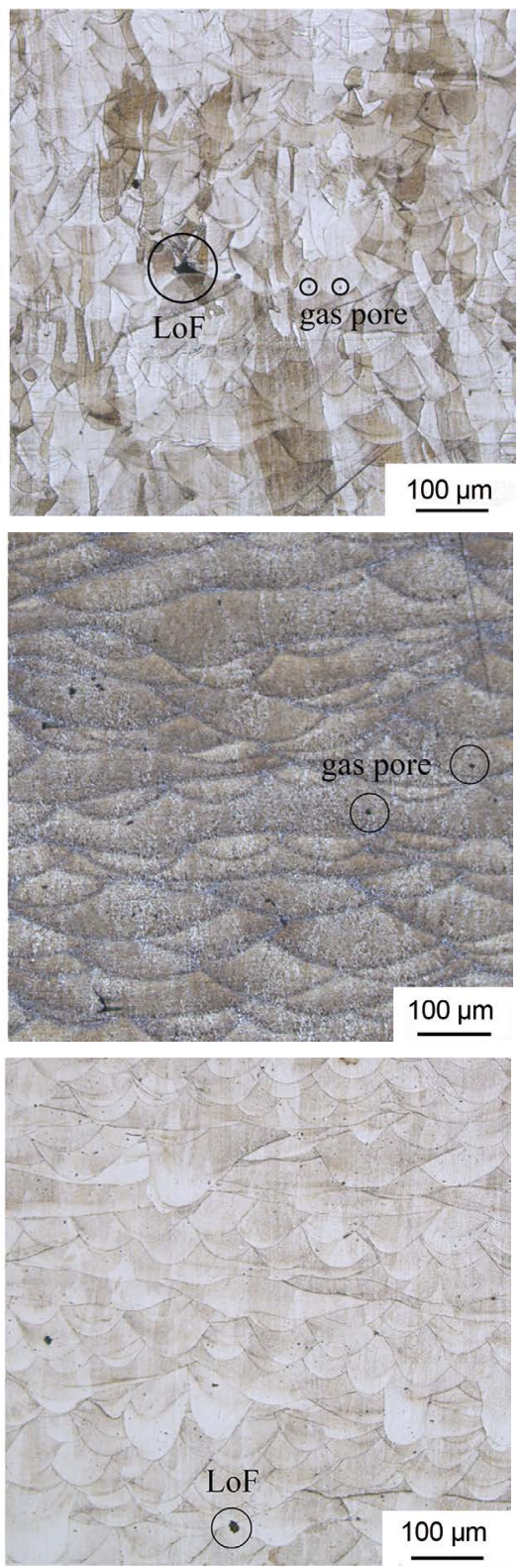

Fig. 3: Lack of fusion, gas pores and keyholes observed in unetched and etched horizontal and vertical sections in three samples: (a) 316L stainless steel; (b) AISi10Mg; (c) Inconel 718

\subsection{Defect distribution characteristics}

\subsubsection{L stainless steel}

Figure 4(a) shows the orthographic views of micro-CT images of the $316 \mathrm{~L}$ sample. It is observed that a large number of defects with a size over $40 \mu \mathrm{m}$ in diameter are formed inside the sample. Typically, the defects aggregate in the center of the sample with the maximal thickness and the height around $10 \mathrm{~mm}$. Moreover, the defect density decreases with the decrease of wall thickness. Figure 4(b) depicts the calculated DAR versus height of four vertical sections with different thicknesses, respectively. The results show that the defect distribution along the building direction approximately reveals a trend of increase at first and then decrease, the defect area ratio reaches maximum when the building height is about $10 \mathrm{~mm}$, which is almost consistent with the micro$\mathrm{CT}$ results. It should be noticed that as the thickness of the section reduces to $3.75 \mathrm{~mm}$, the deviations of the defect area ratio values increase, compared with other thicker sections. Figure 4(c) gives the contour maps of DAR distribution in four horizontal sections located at different heights, respectively. For Sections 1 and 4 located at the bottom and top parts, respectively, a lower density of defects is observed. Typically, the triangular area of Section 4 shows a higher quality with the lowest defect density. However, Sections 2 and 3 located at the central part display a significantly higher level of defect density, especially in the triangular areas, which indicates a greater deterioration in the forming quality. 
(a)

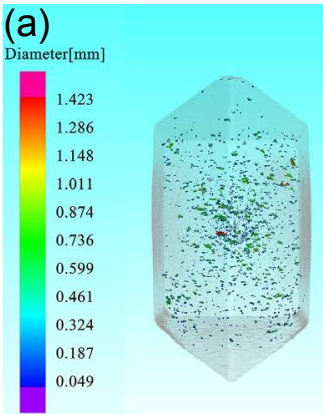

(b)
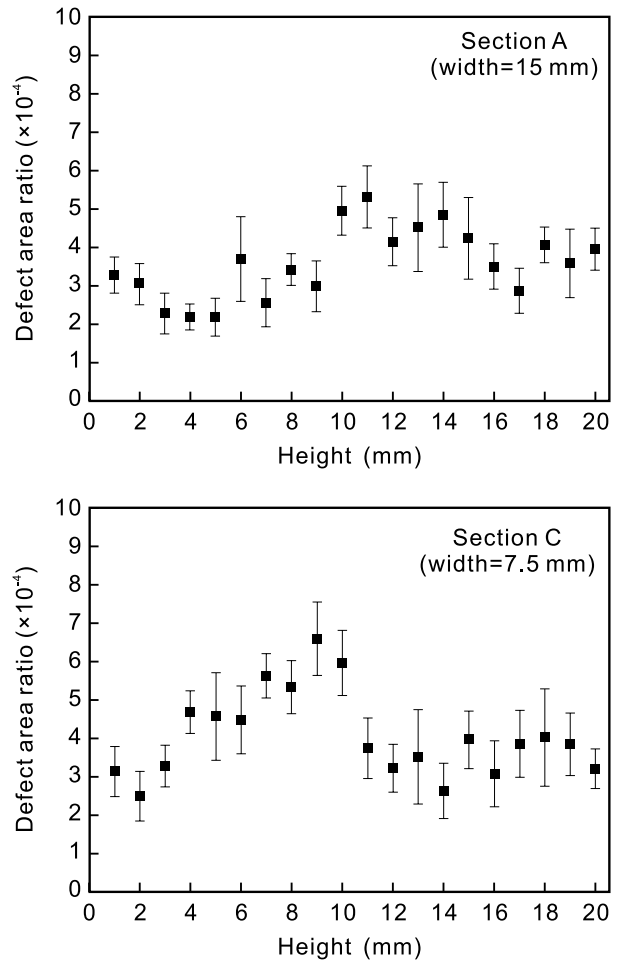

(c)

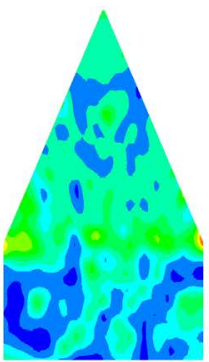

Section 1

$h=4 \mathrm{~mm}$

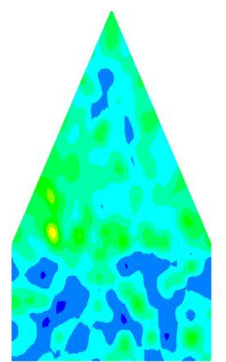

Section 2

$h=8 \mathrm{~mm}$

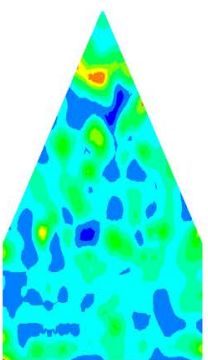

Section 3

$h=12 \mathrm{~mm}$
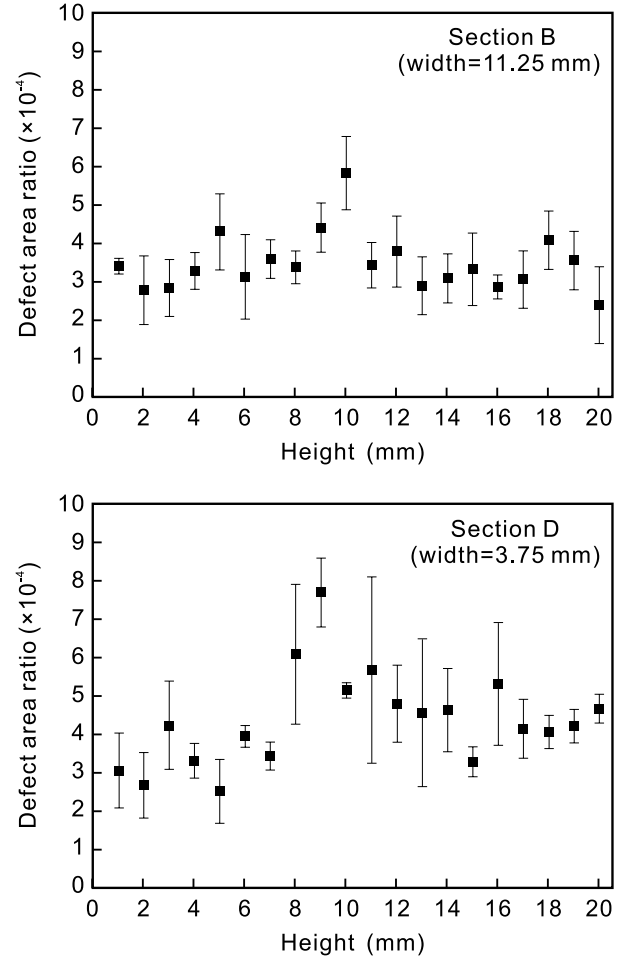

Defect area ratio

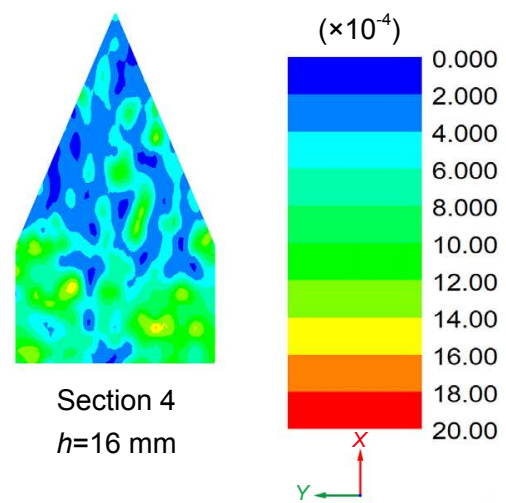

Fig. 4: Micro-CT images of 316L sample (a), scatter diagrams of DAR values in vertical sections of 316L sample (b), and contour maps of DAR values of horizontal sections of $316 \mathrm{~L}$ sample (c)

\subsubsection{AlSi10Mg}

Figure 5(a) shows the orthographic views of micro-CT images of the AlSi10Mg sample. The results reveal that defects with a dimension of 35-480 $\mu \mathrm{m}$ are evenly distributed inside the sample. These are mainly lack of fusions caused by the aluminum oxides generated during the SLM process. Compared with the micro-CT results of the 316L sample, the quantity of defects is large, but the volume is much smaller, which indicates that the relative density of the AlSi10Mg sample is greater than that of the $316 \mathrm{~L}$ sample. In addition, the defect density increases significantly in the area close to the five vertical edges of the sample. From Fig. 5(b), the calculated DAR values tend to increase linearly with the increase of height, but the results of Section D are more discrete than others. It should be noted that these metallographic results are obtained by investigating 
defects smaller than $80 \mu \mathrm{m}$, while the minimum size of defects shown in CT images is $35 \mu \mathrm{m}$, thus a part of defects smaller than $80 \mu \mathrm{m}$ in the CT images are also included in the statistics. With regard to the contour maps of horizontal sections of the AlSi10Mg sample in Fig. 5(c), it is clear that the bottom section has a high forming quality except for the areas near the five angles. With the increase of building height, the probability of defect occurrence grows. The top section has the highest density of defects. It is noted that the areas near the vertical edges show much higher defect density than other areas inside each section without regard to the height, which is consistent with the results of the $\mathrm{CT}$ images.

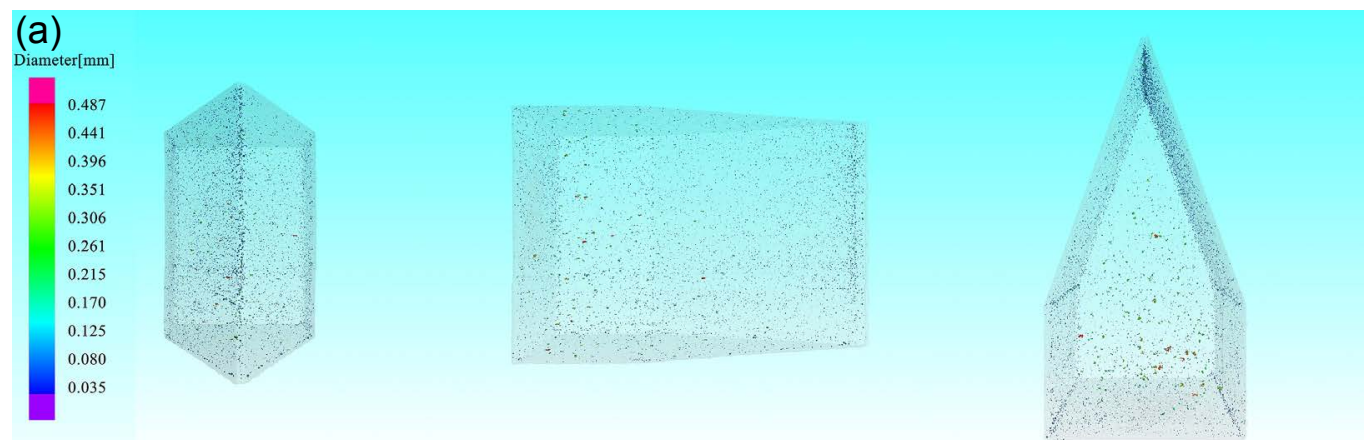

(b)
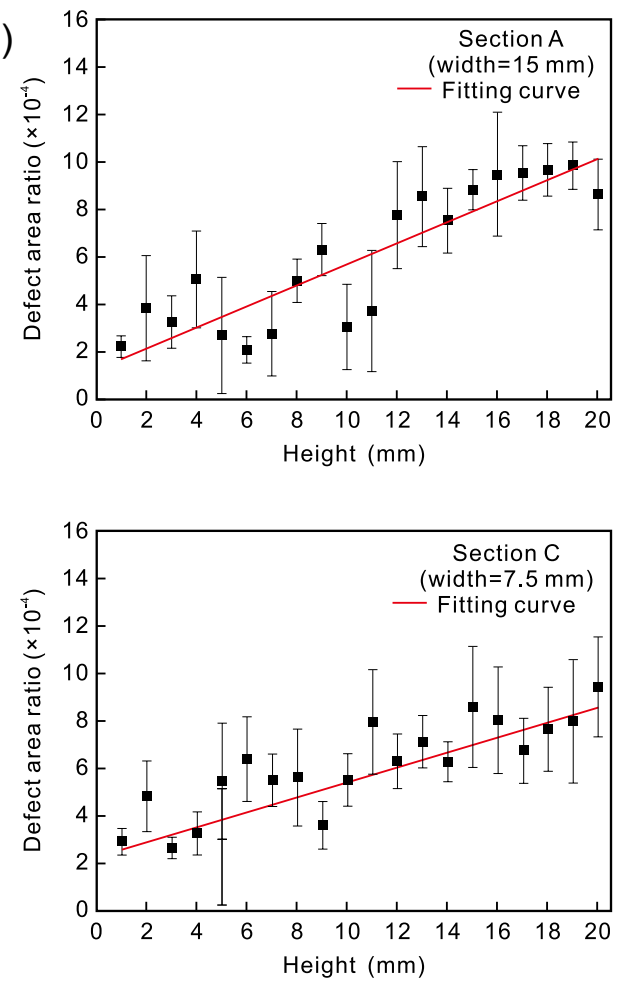

(c)

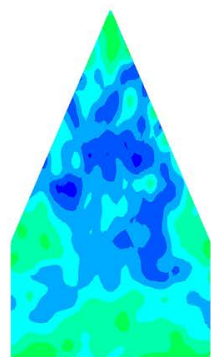

Section 1 $h=4 \mathrm{~mm}$

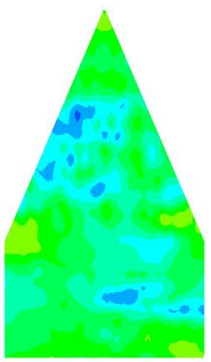

Section 2

$h=8 \mathrm{~mm}$

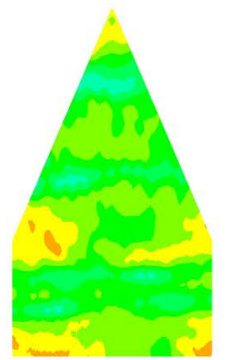

Section 3 $h=12 \mathrm{~mm}$
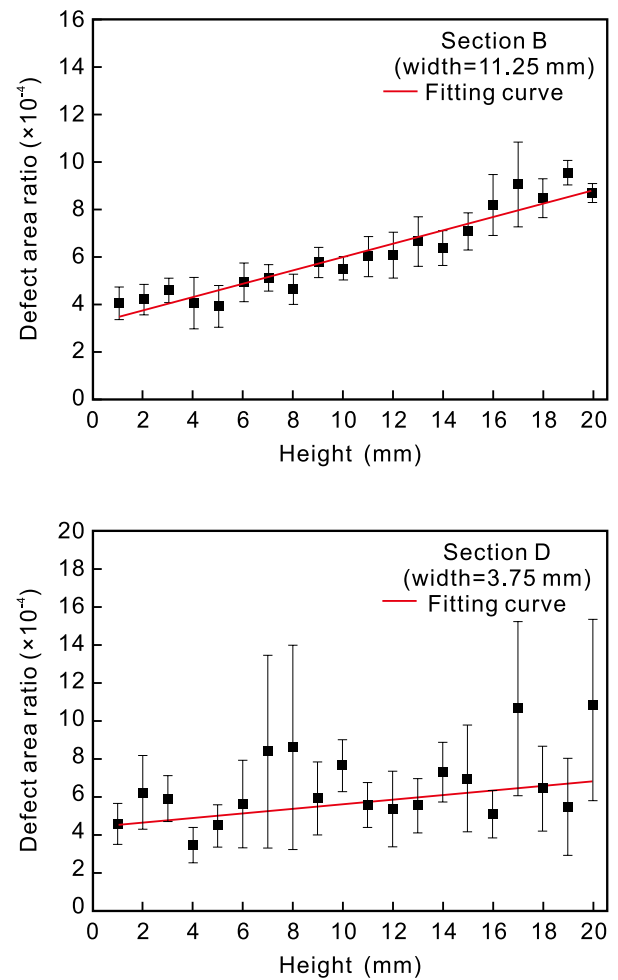

Defect area ratio

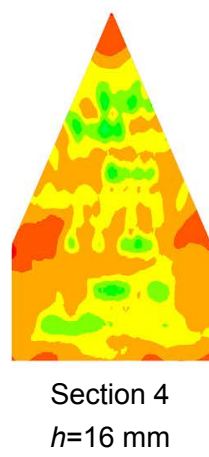

Fig. 5: Micro-CT images of AISi10Mg sample (a), scatter diagrams of DAR values in vertical sections of AISi10Mg sample (b), and contour maps of DAR values of horizontal sections of AISi10Mg sample (c) 


\subsubsection{Inconel 718}

Figure 6(a) shows the orthographic views of micro-CT images of the Inconel 718 sample. The results indicate that most defects are gathered near both ends of the sample while the central part is almost fully dense. Typically, there is a considerable aggregation of defects with an average diameter over $150 \mu \mathrm{m}$ located near the acute angle edge. The scatter diagrams of DAR versus the height of vertical sections of the Inconel 718 sample are shown in Fig. 6(b). It is clear that the defect density is approximately identical regardless of the wall thickness or building height. A similar conclusion can also be obtained with the contour maps of horizontal sections as shown in Fig. 6(c). The fraction of the defect-free area marked as blue is comparable in the inner parts of four sections at different heights. However, the level of defect density located at a corner part, especially areas near the acute and perpendicular edges, exhibits a high defect area ratio in each section.

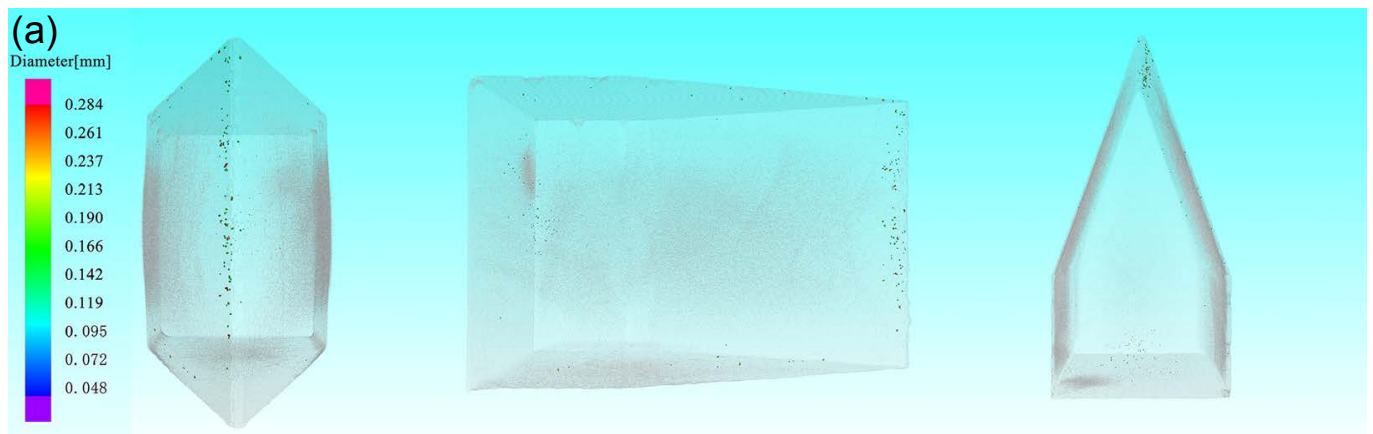

(b)
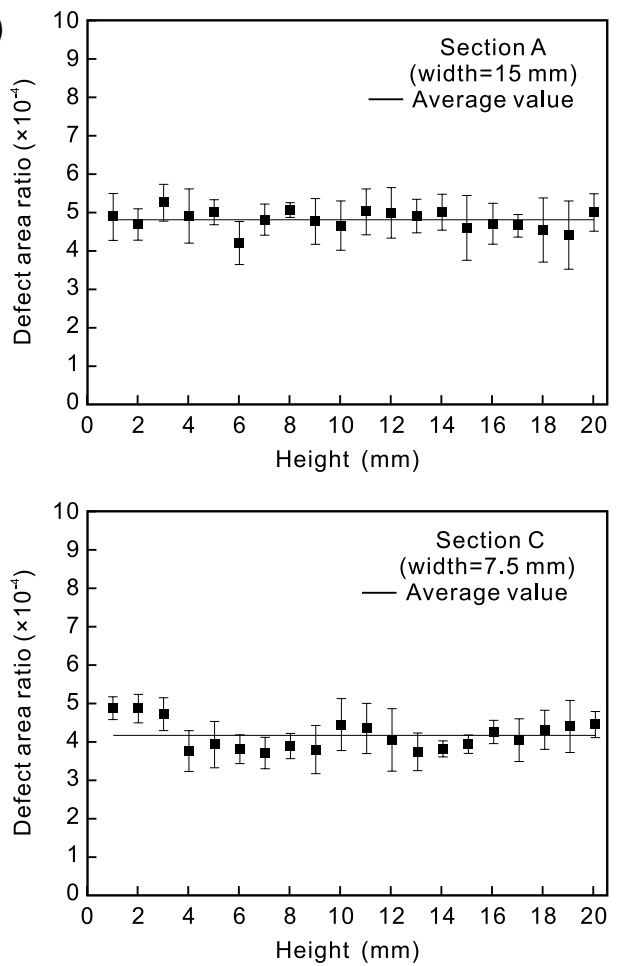

(c)

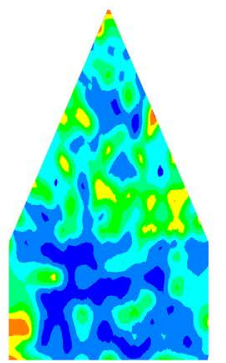

Section 1 $h=4 \mathrm{~mm}$

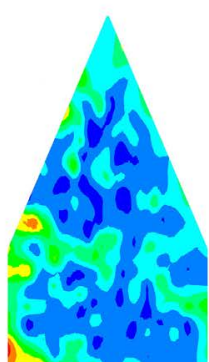

Section 2 $h=8 \mathrm{~mm}$

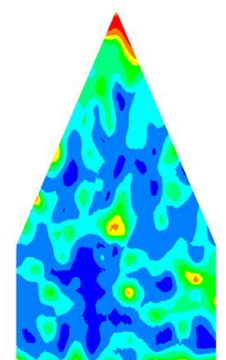

Section 3 $h=12 \mathrm{~mm}$
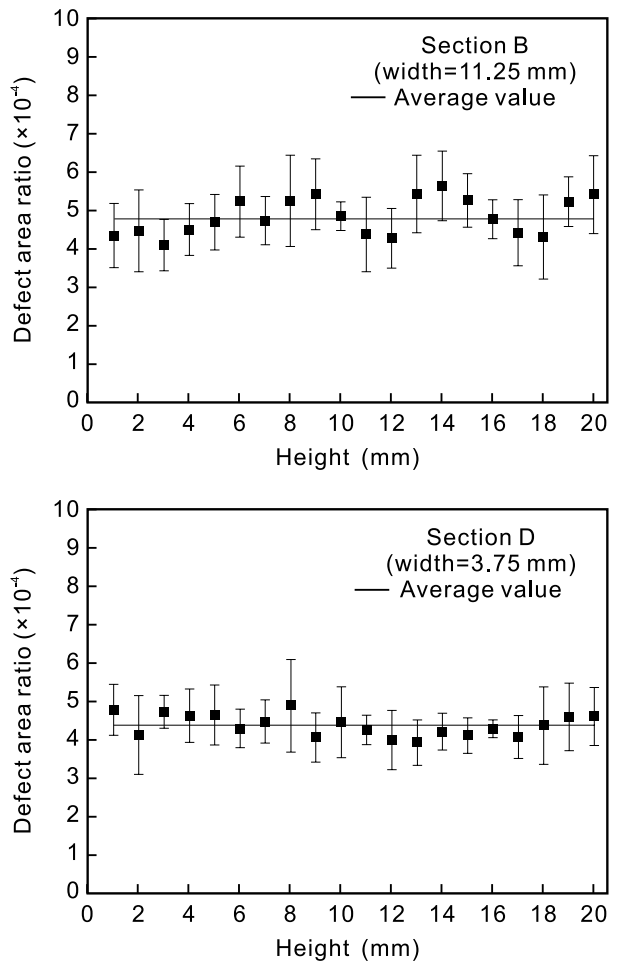

Defect area ratio
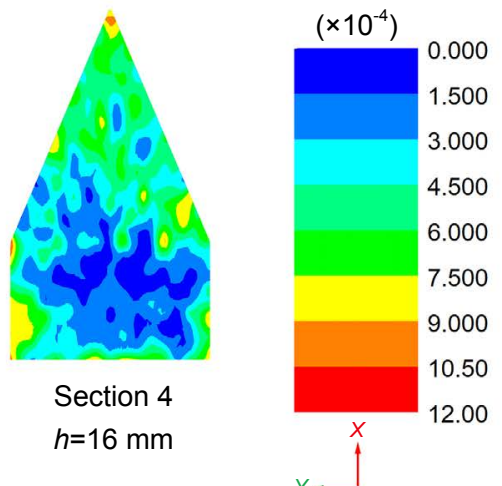

Fig. 6: Micro-CT images of Inconel 718 sample (a), scatter diagrams of DAR values in vertical sections of Inconel 718 sample (b), and contour maps of DAR values of horizontal sections of Inconel 718 sample (c) 


\subsection{Discussion}

Considering the micro-CT and microscopy results, it can be concluded that the defects detected by micro-CT are mainly lack of fusions while defects analyzed by microscopy are mainly gas pores.

As mentioned before, the defect distribution is a reflection of the complex heating-cooling condition during the SLM process. Thus, the distinct defect distribution of the three alloys indicates the different temperature distribution characteristics during the sample preparation process of each alloy. The temperature distribution in the samples during the SLM process is influenced by the energy input of the laser beam and the thermophysical properties of the alloys. Since the energy input is constant and appropriate for each alloy in the present research, the thermophysical properties of the alloys deserve attention. The values of some thermophysical properties of the three alloys at specific temperatures are listed in Table 2.

Table 2: Some thermal properties of the three alloys

$\begin{array}{cccc}\text { Alloy } & \begin{array}{c}\text { Melting point } \\ (\mathrm{K})\end{array} & \begin{array}{c}\text { Thermal conductivity } \\ \left(\mathbf{W} \cdot \mathbf{m}^{-1} \cdot \mathrm{K}^{-1}\right)\end{array} & \begin{array}{c}\text { Viscosity } \\ (\mathbf{m P a} \cdot \mathbf{s})\end{array} \\ \text { 316L stainless steel } & 1,693 & 26(1,300 \mathrm{~K})^{[16]} & 1.4(900 \mathrm{~K})^{[19]} \\ \text { AlSi10Mg } & 869 & 220(750 \mathrm{~K})^{[18]} & 7.12(1,650 \mathrm{~K})^{[21]}\end{array}$

In common, the initial layers at the beginning of the SLM process experience a much higher temperature gradient since a cold substrate is employed to support the component. As a result, the bottom parts of the samples are usually dense with few defects. As the deposition continues, heat accumulation occurs along the scanning direction and building direction ${ }^{[18]}$, which leads to the increase of the maximum temperature and dimension of the molten pool, especially in the thicker parts. For AlSi10Mg, due to the strong hydrogen absorption tendency of the molten pool, gas pores become the main defect type in the AlSi10Mg sample ${ }^{[22]}$. Since the maximum temperature and dimension of the molten pool increase, the solubility and dissolved amount of gas in the molten pool increase, which increase the possibility of gas pore formation. Compared with AlSi10Mg, 316L stainless steel has no tendency of hydrogen absorption, but a small amount of gas still leads to the formation of gas pores. Due to heat accumulation, excessive heat input is hard to avoid, which may result in keyholes, especially in the thicker parts at medium height. Also, the high viscosity and shrinkage tendency of the $316 \mathrm{~L}$ stainless steel molten pool may lead to a decrease in the flowability of the molten pool, which leads to defects ${ }^{[13]}$.

In the later stage of the printing process, because of the high thermal conductivity of AlSi10Mg alloy, the heat transfer in the upper layers is very intense, which leads to a large temperature gradient and as a result, the solidification rate of the molten pool increases ${ }^{[18]}$. Under this circumstance, the solidification will be too rapid for the gas dissolved in the molten pool to escape completely, and then gas pores are formed. As the building height increases, the amount of dissolved gas increases, but the amount of escaping decreases, which leads to the phenomenon in this study that the gas pores increase as the height increases in AlSi10Mg alloy. In the SLM process of the AlSi10Mg sample, since the oxidization reaction occurs continuously, lack of fusions caused by the aluminum oxides are generated and distributed evenly, as investigated in the CT results. For $316 \mathrm{~L}$ stainless steel, the solidification rate in the upper layers decreases because the thermal conductivity of $316 \mathrm{~L}$ is much lower than that of AlSil0Mg, which reduces the formation of gas pores ${ }^{[16]}$. In addition, the viscosity of the molten pool decreases because of the temperature rise of the molten pool caused by heat accumulation, which also reduces the probability of defect forming. In conclusion, the defect density of the $316 \mathrm{~L}$ stainless steel sample will increase at first and then decrease.

Inconel 718 alloy has the best formability among the three alloys. Although the thermophysical properties of Inconel 718 are close to that of $316 \mathrm{~L}$ stainless steel, the key difference between the two alloys is that Inconel 718 is a multiphase precipitation hardening alloy while $316 \mathrm{~L}$ is a single-phase austenitic alloy. In the solidification process of Inconel 718, eutectic reaction takes place and the solidification temperature range is small, which means the fluidity of the molten pool is relatively good and defects are hardly generated ${ }^{[23]}$. In addition, Inconel 718 has little affinity with gas and as a result gas pores are rarely seen inside the Inconel 718 sample. Therefore, even though the heat distribution is similar to that of $316 \mathrm{~L}$ stainless steel, the defect density is lower and remains almost unchanged along the building direction.

Another critical effect on the defect distribution is the geometrical characteristics of components. From the microCT results and contour maps of DAR for horizontal sections, it is clear that the bottom parts of the samples printed initially are quite dense with few defects, which indicates that the process parameters for each alloy are optimized enough to ensure the overall quality. However, in AlSi10Mg and Inconel 718 samples, the defect density located at corners shows an abnormally high level comparing with the inner part. This phenomenon can be explained by two aspects as follows: one is that the scanning pattern, which is normally used as the 
chessboard and/or meander, may cause insufficient padding near the angles in the horizontal plane, leading to the loose overlapping between neighboring layers and/or tracks ${ }^{[24]}$. Another aspect is that the laser beam frequently changes scanning direction as the width gradually narrows to form the angles, thus it is hard to maintain a steady scanning speed especially at the start/end points of the scanning tracks. It is believed that the processing stability is deteriorated and heat is accumulated at these points and defects are easily generated ${ }^{[24]}$. The above mentioned phenomenon is not obvious in the $316 \mathrm{~L}$ sample. In the $316 \mathrm{~L}$ sample, the main type of defect is the lack of fusions caused by high viscosity of the liquid phase and elevated by heat accumulation. Since the scan lines are very short in the near-edge areas, the heat accumulation is rather weak and as a result, defects are not raised significantly ${ }^{[18]}$

The results in this work clearly indicate the defects are easily generated near the corners, thus it should considerably arouse our attention to optimize the geometrical design of the components and the scanning strategy used for the nearedge area. In practice, the edge of SLM components should be re-scanned by an individual pattern to eliminate defects formed near the edge which can improve the forming quality. Furthermore, based on the above discussion, some suggestions on the shape design of metallic parts fabricated by SLM can be put forward. For AlSi10Mg and Inconel 718 alloy, the common suggestion is to avoid too many corners, especially acute angles. If there must be some sharp edges, the edges should be placed in the $X-Y$ plane as much as possible by means of adjusting the building orientation, so as to avoid the edges appearing parallel to the $Z$ axis. Also, the cooling time between two successive layers (inter layer time) in the SLM process can be prolonged properly in order to relieve heat accumulation which would help reduce defects. It should be pointed out that for AlSi10Mg alloy, the height of components in $Z$ direction should be controlled as much as possible through adjusting the building orientation or other means.

\section{Conclusion}

This study presents experimental results related to the defect distribution of $316 \mathrm{~L}$ stainless steel, A1Si10Mg and Inconel 718 samples produced by SLM, considering the effects of geometrical characteristics of the samples with designed geometry. Defects in 316L stainless steel samples tend to be distributed in areas with large wall thickness at medium height of the sample. High viscosity and high tendency of solidification shrinkage are crucial factors for $316 \mathrm{~L}$ stainless steel leading to visible sensitivity to heat accumulation. In AlSi10Mg samples, defects tend to be distributed densely and evenly in the inner part and increase significantly near the vertical edges. Gas pores with small dimensions rise with the building height increases. Inconel 718 alloy, which has a good formability for SLM process, can produce nearly full-density components. Inside the Inconel 718 parts, a few remaining defects tend to be distributed near the vertical edges, which is similar to AlSi10Mg alloy.

\section{Acknowledgements}

This work was supported by the National Key R\&D Program of China (No. 2018YFB1106100) and Jiangsu Key Laboratory for Advanced Metallic Materials (No. BM2007204).

\section{References}

[1] Vartanian K, McDonald T. Accelerating industrial adoption of metal additive manufacturing technology. JOM, 2016, 68(3): 806-810.

[2] DebRoy T, Wei H L, Zuback J S, et al. Additive manufacturing of metallic components - Process, structure and properties. Progress in Materials Science, 2018, 92: 112-224.

[3] Zhang B, Li Y T, Bai Q. Defect formation mechanisms in selective laser melting: A review. Chinese Journal of Mechanical Engineering, 2017, 30(3): 515-527.

[4] Wang H Z, Zou Y. Microscale interaction between laser and metal powder in powder-bed additive manufacturing: Conduction mode versus keyhole mode. International Journal of Heat and Mass Transfer, 2019, 142: 118473.

[5] Snell R, Tammas-Williams S, Chechik L, et al. Methods for rapid pore cassification in metal additive manufacturing. JOM, 2019, 72(1): 101-109.

[6] Vilaro T, Colin C, Bartout J D. As-fabricated and heat-treated microstructures of the Ti-6Al-4V alloy processed by selective laser melting. Metallurgical and Materials Transactions A: Physical Metallurgy and Materials Science, 2011, 42(10): 3190-3199.

[7] Li R D, Liu J H, Shi Y S, et al. Balling behavior of stainless steel and nickel powder during selective laser melting process. The International Journal of Advanced Manufacturing Technology, 2012, 59(9-12): 1025-1035.

[8] Kempen K, Thijs L, Humbeeck J V, et al. Processing AISi10Mg by selective laser melting: Parameter optimisation and material characterisation. Materials Science and Technology, 2015, 31(8): 917-923.

[9] Liverani E, Toschi S, Ceschini L, et al. Effect of selective laser melting (SLM) process parameters on microstructure and mechanical properties of $316 \mathrm{~L}$ austenitic stainless steel. Journal of Materials Processing Technology, 2017, 249: 255-263.

[10] Choi J P, Shin G H, Yang S, et al. Densification and microstructural investigation of Inconel 718 parts fabricated by selective laser melting. Powder Technology, 2017, 310: 60-66.

[11] Nie X J, Zhang H, Zhu H H, et al. Analysis of processing parameters and characteristics of selective laser melted high strength Al-Cu-Mg alloys: From single tracks to cubic samples. Journal of Materials Processing Technology, 2018, 256: 69-77.

[12] Mohr G, Altenburg S J, Hilgenberg K. Effects of inter layer time and build height on resulting properties of $316 \mathrm{~L}$ stainless steel processed by laser powder bed fusion. Additive Manufacturing, 2020, 32: 101080 .

[13] Cherry J A, Davies H M, Mehmood S, et al. Investigation into the effect of process parameters on microstructural and physical properties of $316 \mathrm{~L}$ stainless steel parts by selective laser melting. The International Journal of Advanced Manufacturing Technology, 2015, 76(5-8): 869-879.

[14] Wu H H, Li J F, Wei Z Y, et al. Effect of processing parameters on forming defects during selective laser melting of AISi10Mg powder. Rapid Prototyping Journal, 2020, 26(5): 871-879. 
[15] Weingarten C, Buchbinder D, Pirch N, et al. Formation and reduction of hydrogen porosity during selective laser melting of AISi10Mg. Journal of Materials Processing Technology, 2015, 221: 112-120.

[16] Manvatkar V, De A, Debroy T. Heat transfer and material flow during laser assisted multi-layer additive manufacturing. Journal of Applied Physics, 2014, 116(12): 124905.

[17] Mills K C, Su Y C, Li Z S, et al. Equations for the calculation of the thermo-physical properties of stainless steel. ISIJ International, 2004, 44(10): 1661-1668.

[18] Hu H W, Ding X P, Wang L Z. Numerical analysis of heat transfer during multi-layer selective laser melting of AISi10Mg. Optik, 2016, 127(20): 8883-8891.

[19] Wei P, Wei ZY, Chen Z, et al. Numerical simulation and parametric analysis of selective laser melting process of AlSi10Mg powder. Applied Physics A: Materials Science and Processing, 2017, 123(8): 540.

[20] Zhang P D, Zhang D Y, Liu Z, et al. Microscopic simulation of the effect of process parameters by the Marangoni convection on the temperature field of Inconel 718 alloy using selective laser melting. In: Conference on Advanced Laser Processing and Manufacturing II: Beijing, 2018.
[21] Overfelt R A, Matlock C A, Wells M E. Viscosity of superalloy 718 by the oscillating vessel technique. Metallurgical and Materials Transactions B, 1996, 27(4): 698-701.

[22] Galy C, Guen E L, Lacoste E, et al. Main defects observed in aluminum alloy parts produced by SLM: From causes to consequences. Additive Manufacturing, 2018, 22: 165-175.

[23] Yang $H$ H, Jing G Y, Gao P, et al. Effects of circular beam oscillation technique on formability and solidification behaviour of selective laser melted Inconel 718: From single tracks to cuboid samples. Journal of Materials Science and Technology, 2020, 51: 137-150.

[24] Thijs L, Kempen K, Kruth J P, et al. Fine-structured aluminium products with controllable texture by selective laser melting of pre-alloyed AISi10Mg powder. Acta Materialia, 2013, 61(5): 1809-1819. 